\title{
Author Index for Abstracts
}

$\mathrm{O}=$ Oral presentation, $\mathrm{P}=$ poster presentation

Adamidis,A. P19 Alexander, K. P47, P78, P96 Araujo,R.C. P60

Aydin,A. P32

Bader,B. P60

Bähring, S. P32, P34

Baldamus, C.A. 018

Baltatu, O. P60

Barbey, M. P96

Barenbrock, M. 024, P54, P55, P56, P93

Bäurle,L. PI8

Baur,E. 04

Becker, C. P43

Becker, K. P28

Becker, R.H.A. P10, Pll

Beige, J. P33, P35, P36

Beltran,B. Pill

Block, D. 036, P59

Bochmann, R.P. P63

Bock,C. 012

Bodin,F. P84

Boldt,T. P64

Bölscher,S. P75

Brodde, O.E. P27, P28

Brom,J. P72

Buikema,H. 010

Burchard, M. P62

Busjahn,A. 019, P34, P69

Bychkow,R. P24

Cai,L. P53 Cameron, J.D. P51 Celis, H. PI02 Chan,G. P25 Chao,J. P60 Chung, O. 030

Classen, H.G. P83 Clausmeyer, S. PI Clorius, J.H. 08 Costerousse, O. P5 Creutzig,A. P47, P78, P96 Oilman, J. P4

Dämmrich, J. P82 Dart, A.M. P51.P80 Datté,J.Y. P7 DeMey,J.G.R. P49 Dendorfer,A. P16, P17, P18 Dibona, G.F. P21 Dietrich, V. PI06 Dikow, R. P14

Distler, A. 014, 025, P33, P35, P36, P39,

P40 Dodt,C. 013

Dominiak,P. P16, P17, P18, P23 Donskaya,A. P81 Dörffel,Y. 031 Drab,M. P43 Du,X.J. P51

Dudek,M. 06,020 Düsing,R. O18, P30 
Eckardt,R. P91 Eckert,S. P107, P108 Elam,M. 013 Endemann, D.H. P9 Engler, S. P6

Engmann,W. P99

Fagard,R. PI02

Fahlbusch,R. 05

Faßheber, T. P39

Faulhaber, H.D. P34, P84

Fehm, H.L. 013

Fierlbeck,W. P82

Fischer, T. P71

Fitschen, M. PI7

Flesch,M. P6

Fliser,D. 02, P14

Fornara, P. 023

Francke, S. P38

Frank, H. 05

Franz, I.W. 09, 026, P73, P74, P87, P88,

P103, P104, P105, P106 Franz,W. P5 Fricke, L. 023 Froguel,P. P38 Frölich,J.C. P89

Frye,B. P62 Funke-Kaiser, H. P26

Gaciong,Z. P82 Gallinat, S. P2, P3 Ganten, D. 035, P60 Gatzka,C.D. P51.P80 Geiger, H. 05, P75, P98 Gerdts,E. 019 Gerhard, U. P77 Giessler,C. P28 Giet, M. van der P42 Gilst, W.H. van O10, P6 Gleichmann, U. PI07, PI08 Gohlke,H.R. P34

Gohlke, P. 029, P7 Gollasch, M. P24, P41 Grabensee,B. P79 Greiner,B. PI 10 Gretz,N. 032

Grisk,O. P21 Groh,J. PI 12 Gross, V. P57 Grote,L. P94 Gschwend,S. P44 Günther,A. 017

Haase, H. P41

Hager,J. P38

Hahne, G. P29

Haller,H. 03, 04, 016, P24, P32, P41,

P43, P69 Hans, U. P77 Hartmann, E. 016 Haubeck,H. P85

Hausberg, M. 024, P54, P55, P56, P93 Häuser,W. 034, PI3 Haxelmans, S. P50 Heath, R. P84

Hehr,A. P28 Heidland,A. P82 Heine, O. P65 Heinroth-Hoffmann, I. P27 Heintz, B. P70, P85

Heitmann, J. P94 Hempel, A. 04 Hendricks, P. P90 Hense,H.W. P90, P95 Herdegen, T. P2

Hertling, S. P4 Hertwig, S. 021 Hesse, H.C.H. P63 Heusser,K. PI9 Heyer, C. von P4

Heyl,W. P70 Hilgers, K. 01 Hoehe,M. 019 Hoffmann, S. P60 Hohage, H. 024, P50, P77

Hohenbleicher, H. P12, P35, P36 Holmer, S.R. P95 Homuth,V. 03,04 Hoyer,J. 014, P39, P40, P61 Hübner,N. 035, P53 Hupf,H. 033, P58

\section{KAHGER}

E-Mail karger@karger.ch Fax+ 41613061234 http://www. karger.ch

(C) 1997 S. KargerAG, Basel

351

Пlner, S. 030 Inagami, T. 034 Ives, H.E. P25

Jacobi, J. 01 Jähnichen, G. 030 Jankowsk,J. OH.P46 Jansa,U. P84 Jennings, G.L. P80 John, S. 022 Jöhren,O. 034, PI3 Jüpner, A. 03

Kachel,H.G. P75 Keil,M. PIO,PП Ketelhut, R.G. PI04, PI05 Kettenhofen, R. P30 Keyser,B. 013 Kirchberg, A. 07 Kisters, K. P54, P67, P68, P76 Klan,R. P89 Klingbeil,A. P86

Klingl,K.G. P84 Klostermann, F. P12 Klöting,I. 036 Knoblauch,H. P34 Ko,Y. P30, P31 
Koch, A. PI 10 Köhler,M. 016 Köhler,R. O14, P39, P40 Kollenbaum, V.E. P22, P110 Kollmann,K. 07 Kolloch, R.E. 06, 020, P38 Körner,J. P76 Kosch,M. P54, P56 Kraft, K. Pill Kränzlin,B. 032 Krefting, E.R. P67, P68 Kreutz, R. 035, P33, P36 Krivosikova, Z. P82 Kruse, H.J. P47, P78, P96 Kügelgen, I. von 015 Kutkuhn,B. P79

Lachat,M. P45 Langenfeld, M.R.W. P72 Lätsch,C. 031 Laue,O. 018 Lee,M.A. P53 Lee,Y.A. P53 Leicht,H. P108 Lemmer,B. P70 Lennarz,M. 06, 020, P38 Lenz,T. P75, P98 Leotta,K. 08 Li,G.H. 019 Li,J.S. P9 Liang,Y.L. P51 Lindpaintner, K. 035, P53 Lindschau,C. P41

Linz,W. O28, P10, P11

Lippoldt,A. P57

Lohmann, F.W. P97

Lottermoser, K. P48, P52

Luchner,A. P95

Lüders, S. 07, 027, P91, PI09

Ludwig,M. 06,020,P38

Luft,F.C. 03,04,016, 019, P24, P32,

P34, P41, P43, P57, P69 Lund-Johansen, P. 019, P101 Lüscher,T.F. P45, P92

Makarova, L. P81

Malinski, T. 028

Mann, J.F.E. P84

Martus,P. P86

Maser-Gluth, C. 032

Meffert, S. P3

Meinhardt, R. PI09

Mengden, T. Pill

Menzel, S. 017

Meyden, J. van der 09, P87

Meyer, GJ. P22, P110

Meyer, W. P22

Miethke,A. P41

Mo, R. 019

Morano, I. P41

Motz,W. 021

Mrowka,C. P85

Müller, J.F.M. 026, P73, P74, P88, P103,

P106 Müller-Esterl, W. PI8 Münck,A.C. P8 Muscholl,M. P95

Nagy,Z. P34 Naraghi,R. 05 Nemenoff, R.A. P25 Niederhoffer, N. 012 Niedermayer, W. PI 10 Nobiling, R. P83 Noll, G. P45, P92 Nürnberger, J. P8

Obermüller, N. 032 Oddou-Stock, P. P84 Oliveira, S.M. P60 Omvik,P. O19, P101

Orzechowski, H.D. OП, P26, P44

Paczek,L. P82

Paul, M. O1O, 017, P5, P6, P9, P26, P44,

P61 Pavlova, D. P81 Pees,C. 029, P7 Perekalskaya, M. P81 Persson,P.B. P12, P15

Peschke,B. P75 Pesquero,B. P60 Peter, J.H. P94

Peters, B. 032 Peters, J. 032, PI Philipp,T. O10, P5, P8 Pinto, S.J. O10, P61 Pinto, Y.M. O10, P5, P6 Planitz,C. P63 Plehm,R. P60 Podstufka,T. P32 Pönicke,K. P27 Potthoff,W. Oil

Predel, H.G P65, P66 Prehn,S. 016 Prinz,U. P66 Proeschild, F. P99 Pruys,M. P65 
Qadri,F. P18 Qui,S. P92

Raasch,W. P17, P23

Rahn, H.K. 024, P49, P50, P54, P55, P56,

P67, P68, P76, P77, P93 Rascher,W. P4 Rath,W. P70 Rautenberg, B. 031 Rehn,E.C. P15

Rettig,R. 021,036, P59 Reusch, H.P. P25 Reuter,U. P63 Richter, CM. P26 Richter,N. P66

Ried,C. P24, P41 Riedel, H. P32

Riegger, G.A.J. 033, P58, , P95 Ringel,J. 025,P33 Ritz,E. 02, P14 Rob,P.M. 023,P83

Roessler,U. 08 Ross, A. P98 Rossius,B. 030 Rost, R.E. P65, P66 Roveda, F. P66 Rückert,E.

03 Ruess,C. P46 Ruhwinkel,J. P62 Rump,L.C. 015 Ruschitzka, F. P45

Saavedra, J.M. 034, PI3

Sachinidis, A. 018, P30, P31, P48, P52

Sack, K. 023, P83

Schaefer,F. 02

Schäfers, R.F. P8

Schaper,J. Pll

Schaupp, S. 09, P74, P87, P88

Scheuch,K. P64

Scheuermann, E.H. P75

Schiffrin, E.L. P9

Schlaich, M.P. 01,022, P86

Schlatter,E. P49, P50

352

Author Index

Schlüter,H. 011, P42, P46

Schmid,D. 02

Schmieder, R. 01, 022, PI9, P71, P72,

P86 Schneider, M.P. P71 Schnoor,P. PI 10 Schnoor,P.W. P22

Schobel, H.P. O1, 05, P19, P20, P71, P Schölkens, B.A. O28, P10, PП Scholze,J. O31, P99, P104, P112 Schorr, U. 025, P12, P36 Schottler,T. 08

Schrader,J. 07, 027, P91, P109 Schramm,T. P65, P66 Schrandt,G. 027, P91 Schumm-

Draeger, P. P98 Schunkert, H. 033, P58, P95 Schuster, H. 019, P32, P34 Schwartz, C. P23

Schwarz,B. 07 Sebekova, K. P82 Seewald, S. P30, P31, P48, P52 Seibt,R. P64 Seifert,S. P12

Seul,C. P30, P31 Sharaeva,M. PI00

Sharma, A.M. 025, P12, P33, P35, P37 Shaw, S. P445 Sieberth, H.G. P70, P85 Somers, V.K.

P20 Spieker,C. P55, P67, P68, P76 Spieker,L.E. P92 Spieß,S. 036 Spitznagel, H. 030

Spormann, D. P84 Stachon,A. P50

Staessen, J. PI02

Stauss, H.M. PI5

Stegemann, H. P50

Stein, G. P84

Steinhoff,J. 023

Steinmetz, M. P49

Stichtenoth, D.O. P89

Stock, P. 035

Stöcker, G. P85 
Strauss, M. P32

Stroth, U. P3

Struk, B. 035

Stula, M. P6, P44

Stumpe, K.O. 06, 020, 025, P38

Suwelack,B. P93

Szabo,B. 012

Tepel, M. Oil, P29, P42, P46, P62

Thalhammer, C. P69

Thijs,L. PI02

Tian,X. P5

Timmermann, B. 019

Toka,H. P32

Toka,O. P32

Tönnesmann, U. 09, 026, P73, P74,

P105 Touyz,R.M. P9 Trenkwalder, P. P90 Triebenecker, A. P63 Tschöpe,O. P60

Unger,T. 029, 030, P2, P3, P4, P15

Veelken,R. 022 Veldhuis, J.D. 02, P14 Vereschagina, G. P81

Verges, B. P69

Vetter, H. P30, P31, P48, P52, PI 11

Viskovatykh, M. P81

Voiculescu, A. P79

Völzke,H. V21

Wallukat, G. 03 Walter, C. P69 Weihprecht, H. 022, P72 Weisser,B. P48, P52 Weißñog,E.

P96 Wenzel,R.R. P92 Wieczorek, I. P47, P78, P96 Wiemer,G. O28, P10, Pll Wienker,T.F. 019 Will,D. P22, P110 Willinek, W.A. 06,020 Witta,J. P93 Witte,K. P70 Witte,M. 07,027

Wobus, A. P43 Wolfrum,S. PI6 Woyth,A. 032

Xia,Q. 030

Yakhontov,D. P81 Yu, M.H. P2

Zeidler,H. P89

Zidek, W. Oil, P29, P42, P46, P62, P68,

P76 Ziegler, A. P7 Zimmermann, U. P8 Züchner,C. 027, P91 Zuna,I. 08

Author Index

353 